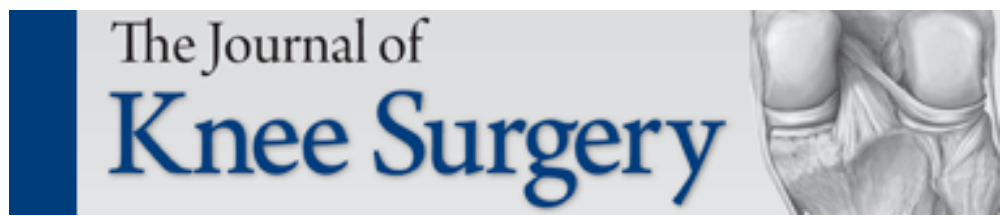

\title{
Primary Stability in Cementless Rotating Platform Total Knee Arthroplasty
}

\begin{tabular}{|c|l|}
\hline Journal: & Journal of Knee Surgery \\
\hline Manuscript ID & Draft \\
\hline Specialty Area: & $\begin{array}{l}\text { total knee arthroplasty, cementless, digital image correlation, } \\
\text { micromotion, mobile bearing }\end{array}$ \\
\hline & $\begin{array}{l}\text { Highly porous ingrowth surfaces have been introduced into tibial tray } \\
\text { fixation to improve long-term survivorship in cementless total knee } \\
\text { arthroplasty. This study was designed to evaluate the effect of a new } \\
\text { generation of porous ingrowth surface on primary stability in the } \\
\text { implanted cementless tibial component. Three tibial tray designs } \\
\text { possessing sintered bead or roughened porous coating ingrowth surfaces } \\
\text { were implanted into a foam tibia model with primary stability assessed } \\
\text { via digital image correlation during stair descent and condylar liftoff } \\
\text { loading. Follow-up testing was conducted by implanting matched pair } \\
\text { cadaveric tibias with otherwise identical trays with two iterations of } \\
\text { ingrowth surface design. Trays were loaded and micromotion evaluated } \\
\text { in a condylar liftoff model. The sintered bead tibial tray exhibited slightly } \\
\text { lower micromotion than the roughened porous coating in stair descent } \\
\text { loading. However, no significant difference in primary stability was } \\
\text { observed in condylar liftoff loading in either foam or cadaveric } \\
\text { specimens. Cementless tibial trays featuring two different iterations of } \\
\text { porous ingrowth surfaces both demonstrated good stability in cadaveric } \\
\text { specimens with less than 80 microns of micromotion and 1 mm of } \\
\text { subsidence under cyclic loading. While improved ingrowth surfaces may } \\
\text { lead to improved biological fixation and long-term osteointegration, this } \\
\text { study was unable to identify a gross improvement in primary stability } \\
\text { associated subsequent ingrown surface design iteration. }\end{array}$ \\
\hline Abstractions \\
\hline
\end{tabular}

\section{SCHOLARONE Manuscripts}




\section{Primary Stability in Cementless Rotating Platform Total Knee Arthroplasty}

3 Abstract: Highly porous ingrowth surfaces have been introduced into tibial tray fixation to

4 improve long-term survivorship in cementless total knee arthroplasty. This study was designed

5 to evaluate the effect of a new generation of porous ingrowth surface on primary stability in

6 the implanted cementless tibial component. Three tibial tray designs possessing sintered bead

7 or roughened porous coating ingrowth surfaces were implanted into a foam tibia model with

8 primary stability assessed via digital image correlation during stair descent and condylar liftoff

9 loading. Follow-up testing was conducted by implanting matched pair cadaveric tibias with

10 otherwise identical trays with two iterations of ingrowth surface design. Trays were loaded and

11 micromotion evaluated in a condylar liftoff model. The sintered bead tibial tray exhibited

12 slightly lower micromotion than the roughened porous coating in stair descent loading.

13 However, no significant difference in primary stability was observed in condylar liftoff loading in

14 either foam or cadaveric specimens. Cementless tibial trays featuring two different iterations of

15 porous ingrowth surfaces both demonstrated good stability in cadaveric specimens with less

16 than 80 microns of micromotion and $1 \mathrm{~mm}$ of subsidence under cyclic loading. While improved

17 ingrowth surfaces may lead to improved biological fixation and long-term osteointegration, this

18 study was unable to identify a gross improvement in primary stability associated subsequent

19 ingrown surface design iteration.

20 Keywords: total knee arthroplasty, cementless, digital image correlation, micromotion, mobile

21 bearing 
Cemented total knee arthroplasty (TKA) remains the standard of care for late-stage

bicompartmental osteoarthritis of the knee across the United States, with more than $88 \%$ of

TKAs utilizing polymethyl-methacrylate bone cement for tibial and femoral component

fixation. ${ }^{1}$ Long-term clinical survivorship in cemented TKA is high, with 20 -plus year follow-up

studies reporting survivorship over $90 \%$ across a variety of component designs and

manufacturers. ${ }^{2-6}$ When clinical failure of cemented TKA components does occur, revision has

been most commonly associated with aseptic loosening, frequently in the tibial component, as

cemented TKA relies on long-term fixation via polymethy-methacrylate bone cement, which can

break down, debond, or migrate over time..$^{7-10}$ In response to the failure mechanisms

32 frequently associated with cemented TKA, cementless TKA has been met with renewed interest over the past decade as a possible means to extend the effective lifespan of TKA, particularly in younger patients. Cementless TKA, which relies on permanent biological fixation for long-term component stability, was originally introduced in adult reconstruction with varying degrees of success. A number of studies have reported positive results with early cementless TKA, with tibial component survivorship reported up to $96.8 \%$ at 20 years follow-up. ${ }^{11-14}$ However, metal backed patellar components, failure of bony ingrowth, tibial component radiolucent lines and osteolytic lesions have led to poor outcomes in other series. ${ }^{15-17}$ studies have demonstrated that bony ingrowth is impeded when bone-implant interface

42 micromotion approaches 150 microns, ${ }^{18}$ and is more likely to occur when early interface 43 micromotion is less than $40-50$ microns. ${ }^{19}$ Seeking to enhance the clinical performance of 
44 cementless TKA devices, manufacturers continue to integrate newer generations of ingrowth

45 substrates into the current TKA market. These porous metal constructs feature increased

46 porosity and surface friction to increase primary stability and promote biological fixation. As

47 new design iterations are introduced, biomechanical assessment is necessary to evaluate their

48 effectiveness in providing improved primary stability for increased long-term clinical efficacy in

49 the standard, primary TKA. Therefore, the current study was designed to evaluate the role of

50 tibial tray geometry and cementless ingrowth substrate on primary component stability.

\section{Methods and Materials}

52 Foam Model Testing

53 Three experimental groups ( $n=10$ per group) were employed in preliminary testing within a

54 foam tibia model (Figure 1). The first group "DuoP” consisted of Size 3 LCS Complete Duofix

55 Porocoat tibial trays (DePuy Orthopaedics, Warsaw, IN) featuring a sintered bead ingrowth

56 surface, a rounded central stem and four auxiliary cylindrical pegs. The second group, "DuoG",

57 had a matching geometry to the first group yet incorporated a roughened porous coating and

58 an unpolished central stem. The final group utilized size 3 LCS Complete MBT tibial trays (DePuy

59 Orthopaedics, Warsaw, IN, USA) with a sintered bead ingrowth surface, a rounded, keeled

60 central stem and no auxiliary pegs. All three experimental groups integrated a $10 \mathrm{~mm}$ LCS

61 rotating platform polyethylene bearing (DePuy Orthopaedics, Warsaw, IN, USA).

62 Tibial components were implanted into custom-manufactured foam tibiae using standard

63 instrumentation and manual impaction techniques until the tibial tray had advanced to full

64 contact with the tibial plateau. The bespoke model was based on a standardized geometry 
65 (Model 3401, Pacific Research Laboratories, Vashon) with plateau matching that of a 7 degree posteriorly sloped resection plane and incorporating a $2.5 \mathrm{~mm}$ thick cortical layer $(0.64 \mathrm{~g} / \mathrm{cc}$ closed polyurethane foam) and an inner cancellous layer ( $0.20 \mathrm{~g} / \mathrm{cc}$ open-cell polyurethane foam) (Figure 2). A square distal base was incorporated for ease of fixturing.

Mechanical testing using stair descent and condylar liftoff loading regimens were performed on a biaxial, electrodynamic materials testing frame (ElectroPuls E10,000 A/T, Instron, Norwood, MA) with specimens distally mounted onto a free-translating baseplate. (Figure 3) In stair descent loading, a modified left femoral component (LCS STD, DePuy Orthopaedics) was integrated into the upper grip of the testing frame to apply axial loads at full knee extension with a 60-40 medial-lateral condylar distribution, as verified prior to each test using a contact pressure sensor (K-Scan 4000, Tekscan, Natick, MA). Instrumented TKA axial knee joint loading data during stair descent at 4-months post-op was acquired from a public database (orthoload.com, patient $\mathrm{K} 1 \mathrm{~L}$ ) and used as the axial compression loading profile. A cyclic rotational loading profile from $-1^{\circ}$ to $6.7^{\circ}$ internal rotation closely matching previously published data and micromotion investigations was used to direct rotation-controlled torsional loading. ${ }^{20,21}$ Axial and torsional stair descent loads were applied at $0.75 \mathrm{~Hz}$ for 5000 cycles.

\section{Digital image correlation (DIC) was used to enable high precision, non-contact micromotion} analysis closely following a previously published methodology. ${ }^{22}$ Tibial trays and foam models were prepared for DIC testing by applying a white basecoat and black speckled paint circumferentially around the component rim and cortical surface. Speckle size and density was tuned to DIC manufacturer specifications (GOM Inc., Braunshweig, Germany). A pair of digital cameras were calibrated to a $160 \times 135 \times 120 \mathrm{~mm}$ field of view. During each loading sequence, 
87 a series of paired images was captured at $15 \mathrm{~Hz}$. Regions of interest (ROI) were established in

88

89

90

91

92

93 anterior, medial, lateral, posterolateral and posteromedial areas of the tibial plateau. At each

$\mathrm{ROI}$, a point on the tray rim was paired with a corresponding point on the tibial cortex at an average gage length of $8 \mathrm{~mm}$. To calculate micromotion of the tibial tray at the ROI, the

Euclidean change in peak-to-peak distance between the two points was extracted during post-

processing image analysis. Micromotion data was collected around the entire tray periphery between loading cycles 5000 and 5100.

Secondary testing was completed to create a model for worst-case-scenario liftoff in both medial and lateral condyles. A $28 \mathrm{~mm}$ diameter metal sphere was used to apply a cyclic axial load from 115 to $1500 \mathrm{~N}$ for 150 cycles at $2 \mathrm{~Hz}$, first to the medial, and then to the lateral condyle. ${ }^{23}$ DIC analysis was conducted on the $150^{\text {th }}$ cycle for each liftoff loading model.

To address the repeated measurements taken on the bone specimens during this first phase, statistical analysis was carried out using generalized estimating equations to fit a marginal linear model for mean micromotion response. In addition to considering the type of tibial component implanted, the model was adjusted for measurement region and allowed the effect of each device to depend on the measurement region. Devices were compared within each region after 5000 loading cycles in stair descent testing, and 150 loading cycles in liftoff testing, and if there was evidence of difference within a measurement region, pairwise comparisons were made from asymptotic Wald tests.

Condylar Liftoff in a Cadaveric Model 
107 The second phase of this study was designed to evaluate tibial component primary stability

108 within a cadaveric tibia model, while isolating porous coating design as the independent

109 variable of interest (Figure 4). Two experimental groups were included in this study: the "DuoP"

110 and "DuoG" tibial tray designs, as introduced above. Thirteen fresh-frozen cadaveric tibia pairs

$111(3 / 10 \mathrm{M} / \mathrm{F}, 70.8 \pm$ years, $36.5 \pm 5.3 \mathrm{BMI})$ were acquired from a national tissue bank and stored

112 at $-20^{\circ} \mathrm{C}$. Per implant sizing guidelines, tissue donors were restricted to heights between 157.5

113 to $167.5 \mathrm{~cm}$ for males, and $162.5 \mathrm{~cm}$ to $172.5 \mathrm{~cm}$ for females, in order for all tibias to accept

114 "Size 3" tibial trays. Following qualitative bone quality assessment and size match verification

115 by the implanting orthopaedic surgeon, three specimen pairs were eliminated from testing,

116 leaving 10 tibias per experimental group. Tibial tray assignment was randomized between right

117 and left tibias for the ten matched pair specimens. Tibial resection was targeted at $10 \mathrm{~mm}$ from

118 the high side of the tibia with component alignment targeted at 3 degrees of posterior slope

119 and 3 degrees of anatomic varus using intramedullary instrumentation by a board-certified

120 orthopaedic surgeon. Immediately following specimen preparation, tibias were distally potted

121 in $80 \mathrm{~mm}$ of fast curing polyester resin.

122 Prior to final component insertion and mechanical testing, CT images were collected of all

123 potted, thawed tibial specimens for quantitative bone quality assessment. Tibias were

124 submerged in a saline bath within a vacuum chamber under a $-650 \mathrm{~mm} \mathrm{Hg}$ vacuum, maintained

125 for two minutes and repeated four times to minimize air artifacts within the imaging data.

126 Tibias were then scanned on a 64-slice CT scanner (Optima CT660, GE Healthcare, Waukesha,

127 WI) with scan settings of $120 \mathrm{kV}, 250 \mathrm{~mA}$, slice thickness of $0.6 \mathrm{~mm}$ with no overlap. Apparent

128 bone density $\left(\mathrm{kg} / \mathrm{m}^{3}\right)$ was calculated using the MIMICS 16.0 imaging software (Materialise, 
129 Leuven, Belgium). Four measurement regions of approximately $250 \mathrm{~mm}^{3}$ were defined in 130 anterior, posterior, medial and lateral cancellous bone, positioned between peg hole locations 131 within the first $5 \mathrm{~mm}$ of the proximal tibia distal to the resection surface. Hounsfield units (HU) 132 were linearly scaled relative to an internal phantom and apparent bone density ( $\rho)$ was 133 calculated as follows ${ }^{24}$ :

$$
\rho=0.916 * \mathrm{HU}+114
$$

Following CT scanning, tibial components were inserted into the appropriate cadaveric specimen via manual impaction until full seating of the tray was visually confirmed around the

137 entire tray rim.

Mechanical Testing

Mechanical testing was conducted in a worst-case simulation modelling anteromedial bearing dwell point of the polyethylene bearing, with the bearing positioned in 20 degrees of external rotation, resulting in a posteromedial load point. An axial load of $450 \mathrm{~N}$ at a rate of 1

$144 \mathrm{~Hz}$ was applied for 3000 cycles. During testing, a saline solution was sprayed onto the tibia 145 surface to maintain tissue moisture. Relative micromotion between the tray rim and the 146 cortical bone in the anteromedial tibia was collected.

147 Statistical analysis in the cadaveric phase of this study was designed with the primary aim to 148 identify, independent of variation in tibial specimen bone density, any difference in component micromotion between the two tibial tray designs. To account for the correlation between 
150 specimens of the same donor in the matched pair trials, a mixed effects linear model (a

151 generalization of a random effects ANOVA) was used. Tibial tray design and specimen bone

152 density were set as fixed effects and individual test specimens were set as the random effect.

153 Due to variability changing across tray design and tibial specimens, bootstrapping was used to

154 generate all confidence intervals and p-values for comparisons. All comparisons were based

155 upon data collected at the $3000^{\text {th }}$ loading cycle. Data is reported throughout the study as mean

156 micromotion $\pm 95 \% \mathrm{Cl})$.

\section{Results}

158 Foam Tibia Testing

159 Figure 5 summarizes the differences between device designs within each measurement region 160 after 5000 cycles of stair descent loading. The greatest mean peak micromotion was observed 161 in the DuoG tibial trays, with micromotion ranging from $0.121 \pm 0.018 \mathrm{~mm}$ to $0.191 \pm 0.014$ $162 \mathrm{~mm}$. Micromotion in the DuoP trays ranged from $0.116 \pm 0.011 \mathrm{~mm}$ to $0.165 \pm 0.011 \mathrm{~mm}$, and 163 from $0.099 \pm 0.020 \mathrm{~mm}$ to $0.162 \pm 0.009 \mathrm{~mm}$ in the MBT tibial trays. Micromotion response 164 between the tibial tray and cortical bone differed between the device designs within the 165 anterior $(p=0.036)$ and lateral $(p=0.008)$ regions. Within the anterior measurement region, 166 the DuoP tibial tray produced less micromotion compared to the DuoG design $(p=0.017)$ 167 however, an overall difference in primary stability between the DuoG and MBT designs was not 168 detected $(p=0.746)$. Within the lateral region, the DuoP and MBT designs respond similarly $(p=$ $1690.685)$, and both result in less micromotion compared to the DuoG experimental cohort $(p=$ 1700.002 and $p=0.002$, respectively). There is no evidence that the micromotion differs between 
171 experimental groups in the medial $(p=0.285)$, posteromedial $(p=0.701)$ or posterolateral $(p=$ $1720.010)$ regions.

173 Figure 6 summarizes the differences between the device designs within each region and loading 174 location within the foam liftoff model. The greatest micromotion was observed in the medial 175 measurement region of all three tray designs during medial condylar loading. In that region, the 176 DuoG, DuoP and MBT tray designs demonstrated mean peak micromotions of $0.128 \pm 0.015$ $177 \mathrm{~mm}, 0.120 \pm 0.021 \mathrm{~mm}$, and $0.127 \pm 0.021 \mathrm{~mm}$ respectively. There was no statistical evidence 178 that the effect of the device designs on the micromotion differed across the two measurement 179 regions considered $(p=0.659)$. Similarly, there is no evidence that the effect of tray design on 180 the micromotion differed across the two loading locations $(p=0.657)$.

181 Cadaveric Testing

182 Matched pair tibial specimens showed good consistency in apparent bone density between 183 right and left sides, with an average difference between sides of $0.025 \mathrm{~g} / \mathrm{cm}^{3}$. Apparent tibial 184 bone density was similar between two test groups, ranging between 0.182 and $0.349 \mathrm{~g} / \mathrm{cm}^{3}$ in 185 tibias implanted with DuoP trays, and between 0.185 and $0.349 \mathrm{~g} / \mathrm{cm}^{3}$ in tibias implanted with 186 Duog tibial trays. The data demonstrated the trend of decreased micromotion with increased 187 apparent tibial bone density in both tray designs $(p=0.022)$ with an overall mean decrease in 188 micromotion of $0.0018 \pm 0.0011 \mathrm{~mm}$ for every $0.1 \mathrm{~g} / \mathrm{cm}^{3}$ increase in apparent bone density 189 (Figure 7).

190 Overall micromotion results for the DuoP and DuoG tibial tray designs tested in the cadaveric 191 loading model are presented in Figure 7. Peak micromotion at any point in time during testing 192 ranged between 0.020 and $0.074 \mathrm{~mm}$ in the DuoG tray design and between 0.022 and 0.079 
$193 \mathrm{~mm}$ in the DuoP design. When examining the effect of tray design on tibial component

194 micromotion in the cadaveric model, statistical analysis did not reveal a significant difference in

195 primary stability of the implanted component $(p=0.662)$. Likewise, overall tibial component

196 subsidence after 3000 loading cycles was minimal, with a peak average subsidence of $0.67 \mathrm{~mm}$

197 in the DuoG implanted tibias and $0.91 \mathrm{~mm}$ of subsidence in the DuoP implanted tibias. No

198 statistically significant difference between the two cohorts was identified $(p=0.264)$.

\section{Discussion}

200

201

202

203

204

205

206

207

208

209

210

211

212

213

The current study was designed to evaluate primary stability of the cementless tibial tray based on component geometry and ingrowth surface material design. In this study, when direct liftoff loading was employed in foam models, we observed no overall difference in primary stability between any of the tray designs. Within a more complex stair descent loading of a foam model, tibial tray geometry and ingrowth surface played only a small role in changing the primary stability of the implanted tibial tray. While small differences in interface micromotion were detected between the two generations of porous coating at the anterior and lateral aspects of the tibial plateau, those differences were too small to deem as clinically significant, particularly when based on a testing in a foam surrogate.

In a prior series of studies investigating primary stability of the cementless TKA tibial tray, Bhimji and Meneghini ${ }^{21}$ also found differences in micromotion based on tray design to be limited to the anterior and lateral aspects of the tibia during stair descent loading. In that study, the authors compared pegged and keeled tibial trays, with pegged trays allowing four times greater micromotion along the longitudinal plane anteriorly, and three times greater 
214 longitudinal micromotion laterally. ${ }^{21}$ However, overall mean micromotions reported by the

215 authors in half of the reported instances during stair descent loading range between 300 and

216750 microns, far exceeding the 150 micron expected limit of micromotion enabling bone

217 ingrowth in several measurement regions. Because of the tendency of foam tibia models to

218 overestimate micromotion based on expected clinically relevant primary stability, additional

219 testing within a cadaveric model is necessitated. Historically, the utilization of cadaveric tibial

220 specimens has been the gold standard for orthopaedic device biomechanical research.

221 Biological tissue specimens are inherently highly variable and present some distinct challenges

222 within the research setting, however, the use of native tibial bone stock in the assessment of

223 TKA component stability offers the closest representation to the in vivo post-op TKA. In the

224 current study, we chose to load the cadaveric specimens in a loading position that would match

225 the bending moment across the tibial tray corresponding to the highest micromotion in a

226 previous computational finite element analysis of the same tibial component geometry. ${ }^{25}$

227 During cadaveric testing, we observed a significant trend in increased micromotion based on

228 decreasing measured apparent proximal tibia bone density. This significant increase in

229 micromotion with a decrease in apparent bone density lends to the integrity of the

230 micromotion data and supports the need for caution when selecting patients with poor bone

231 stone for cementless total knee arthroplasty. When comparing primary stability between the

232 two generations of porous ingrowth surfaces within the cadaveric test specimens, micromotion

233 of the implant during dynamic testing fell between 0.020 and $0.072 \mathrm{~mm}$ (20 and 75 microns).

234 While in vivo mechanical loading in far more complex than the loading model currently

235 undertaken, the scale of micromotion response observed in more clinically accurate than the 
236 foam model, and reflects a range in mechanical response expected for suitable primary fixation

237 enabling subsequent, permanent biological fixation in both sets of tibial components.

238 Aseptic loosening of the tibial component remains a primary failure mechanism for cemented

239 total knee arthroplasty. ${ }^{6,26,27}$ The quality of the bone-cement interface has been shown to

240 degrade over time ${ }^{7}$ which is a primary concern for cemented TKA failure associated with

241 component loosening and third-body wear. Consequently, cementless tibial trays should offer

242 improved component stability and reduced risk of aseptic loosening within the target patient

243 demographic to justify the added implant cost and to serve as an effective alternative to the

244 cemented tibial tray. ${ }^{28} \mathrm{~A}$ recent meta-analysis of a porous tantalum monoblock tibia compared

245 with a cemented modular tray concluded that the cementless tray offered improved functional

246 scores, fewer radiolucencies and decreased operating time versus the cemented tray, but could

247 not conclude any overall advantage to the cementless tray at 5-years follow-up. ${ }^{29}$ De Martino et

248 al. ${ }^{30}$ recently published a 10 -year follow-up of porous tantalum monoblock tibial components

249 with no tibial component revisions due to aseptic loosening or migration in 33 knees. Some

250 studies have reported early subsidence trays and medial tibial collapse as a cause for concern in

251 cementless tibial, ${ }^{31}$ while others have reported early implant stability and equivalent

252 performance when compared to cemented TKA. ${ }^{32-34}$ Overall, early reports of cementless TKA

253 with improved porous titanium and tantalum substrates have been favorable when compared

254 to the early cementless TKA designs. ${ }^{17,31,35}$ However, some instances of early aseptic loosening

255 in cementless tibial components persist. ${ }^{36}$

256 In the interest of investigating aseptic loosening in cementless tibial trays, several previous

257 studies have been undertaken to assess the mechanical stability of an implanted tibial tray 
258 through bench testing and finite element analysis. ${ }^{21,22,44,45,23,37-43}$ Many of these studies have 259 used simplified loading models to assess tibial tray stability, while a few more recent studies 260 have included more complex loading scenarios. ${ }^{25,37}$ More recently, radiostereometric analysis

261 has been paired with mechanical testing to evaluate cementless tibial component subsidence in 262 cadaveric models, with median subsidence reported between 0.5 to $2.5 \mathrm{~mm} \cdot{ }^{40,46}$

263 Comparatively, we observed component subsidence of less than $1.0 \mathrm{~mm}$ for the majority of

264 cadaveric specimens tested, though at a lower cycle count than the previous studies.

265 Finite element analyses of tibial micromotion allow for rapid variation of experimental 266 parameters including device design, loading mechanism, and tibial geometry and bone density.

267 Most pertinent to the current study, Taylor et al. ${ }^{25}$ compared micromotion of the tibial plateau

268 across three separate tibial tray designs, including two designs used in the current study. Using 269 a wide range loading patterns simulating activities of daily living, the authors observed 270 maximum micromotions between $0.158 \mathrm{~mm}$ to $0.249 \mathrm{~mm}$, with peak micromotions occurring 271 during swing phase, when stabilizing axial forces are at their lowest. In finite element modelling 272 of stair descent loading, a tibial tray with keeled central stem and no auxiliary pegs (analogous 273 to the MBT experimental group in the current study) exhibited a mean $0.071 \mathrm{~mm}$ and maximum

$2740.211 \mathrm{~mm}$ micromotion across the surface of the tibial plateau. ${ }^{25}$ In the current study, we

275 observed a maximum of $0.162 \pm 0.009 \mathrm{~mm}$ of peripheral micromotion in the same tray design

276 during stair descent loading within the foam model. Similarly, Taylor et al ${ }^{25}$ observed a mean

$2770.069 \mathrm{~mm}$ and a maximum of $0.249 \mathrm{~mm}$ micromotion during stair descent loading in $\mathrm{FE}$

278 modelling of a tray with rounded central stem and four conical pegs (analogous to the DuoP 
279 group in the current study), compared to $0.165 \pm 0.011 \mathrm{~mm}$ peak peripheral micromotion 280 observed in stair descent foam tests.

281 The authors acknowledge limitations associated with the methodology of this study. Both 282 phases of this study incorporate simplified loading conditions which do not precisely replicate 283 the complex musculoskeletal interactions and kinematic properties of the reconstructed knee. 284 The stair descent loading model utilizes partial data collected from an instrumented TKA 285 patient, however, limitations in testing capabilities prohibited the inclusion of anterior-

286 posterior loading detected during patient gait. Furthermore, the native knee undergoes a wide 287 range of activities of daily life which cannot be fully encompassed by solely stair descent and 288 liftoff loading models. An extensive range of loading patterns should be explored for a complete 289 understanding of the mechanisms related to tibial tray primary stability. For reasons of 290 specimen preservation, micromotion measurements within the cadaveric test specimens were 291 limited to a small region of interest over a limited number of loading cycles. Manufactured 292 foam models have been used in a handful of prior studies $21,37,43,47$ as a means of testing primary 293 stability of cementless tibial components, however, open-celled polyurethane foam has not 294 been validated as a representative substitute for tibial cancellous bone. In this study foam tibia 295 models were used as a first step comparison in a standardized test bed, prior to cadaveric 296 testing.

297 In summary, the primary aim of the current study was to determine the difference in primary 298 stability of a rotating platform TKA tibial tray as a function of tray geometry and porous coating 299 design. Similar conclusions were drawn based on testing in foam and native tissues; with 300 minimal overall difference in interface micromotion between the tray designs and ingrowth 
301 surfaces examined. Mechanical testing within the more physiological cadaveric model resulted

302 in more clinically relevant micromotion data, and remains the standard means of comparison

303 when evaluating cementless arthroplasty components. To more fully evaluate the efficacy of

304 current cementless tibial trays, further examination and long-term clinical follow-up is needed.

\section{References}

1. Nguyen LCL, Lehil MS, Bozic KJ. Trends in Total Knee Arthroplasty Implant Utilization. J Arthroplasty. 2015;30(5):739-742. doi:10.1016/j.arth.2014.12.009

2. Diduch DR, Insall JN, Scott WN, Scuderi GR, Font-Rodriguez D. Total knee replacement in young, active patients. J Bone Jt Surg. 1997;79(4):575. doi:10.1016/S00219355(14)74376-0

3. Dixon MC, Brown RR, Parsch D, Scott RD. Modular fixed-bearing total knee arthroplasty with retention of the posterior cruciate ligament: A study of patients followed for a minimum of fifteen years. J Bone Jt Surg - Ser A. 2005;87(3):598-603. doi:10.2106/JBJS.C.00591

4. Ma HM, Lu YC, Ho FY, Huang CH. Long-term results of total condylar knee arthroplasty. J Arthroplasty. 2005;20(5):580-584. doi:10.1016/j.arth.2005.04.006

5. Pavone V, Boettner F, Fickert S, Sculco TP. Total condylar knee arthroplasty: a long-term followup. Clin Orthop Relat Res. 2001;(388):18-25. doi:10.1097/00003086-20010700000005

6. Ritter MA, Keating EM, Sueyoshi T, Davis KE, Barrington JW, Emerson RH. Twenty-FiveYears and Greater, Results After Nonmodular Cemented Total Knee Arthroplasty. J Arthroplasty. 2016;31(10):2199-2202. doi:10.1016/j.arth.2016.01.043

7. Miller MA, Goodheart JR, Izant TH, Rimnac CM, Cleary RJ, Mann KA. Loss of cement-bone interlock in retrieved tibial components from total knee arthroplasties basic research. Clin Orthop Relat Res. 2014;472(1):304-313. doi:10.1007/s11999-013-3248-4

8. Nilsson KG, Kärrholm J, Carlsson L, Dalén T. Hydroxyapatite coating versus cemented fixation of the tibial component in total knee arthroplasty: Prospective randomized comparison of hydroxyapatite- coated and cemented tibial components with 5-year follow-up using radiostereometry. J Arthroplasty. 1999;14(1):9-20. doi:10.1016/S08835403(99)90196-1

9. Nilsson KG, Kärrholm J, Ekelund L, Magnusson P. Evaluation of micromotion in cemented vs uncemented knee arthroplasty in osteoarthrosis and rheumatoid arthritis: Randomized study using roentgen stereophotogrammetric analysis. J Arthroplasty. 1991;6(3):265-278. doi:10.1016/S0883-5403(06)80174-9 
10. Nilsson KG, Karrholm J. Increased varus-valgus tilting of screw-fixated knee prosthesis. J Arthroplasty. 1993;8(5):529-540.

11. Eriksen J, Christensen J, Solgaard S, Schroøder H. The cementless AGC 2000 knee prosthesis: 20-year results in a consecutive series. Acta Orthop Belg. 2009;75(2):225-233.

12. Melton JTK, Mayahi R, Baxter SE, Facek M, Glezos C. Long-term outcome in an uncemented, hydroxyapatite-coated total knee replacement: A 15- to 18-year survivorship analysis. Bone Joint J. 2012;94-B(8):1067-1070. doi:10.1302/0301620X.94B8.28350

13. Watanabe H, Akizuki S, Takizawa T. Survival analysis of a cementless, cruciate-retaining total knee arthroplasty. J Bone Jt Surg. 2004;86(6):824-829. doi:10.1302/0301620X.86B6.15005

14. Ritter MA, Meneghini RM. Twenty-year survivorship of cementless anatomic graduated component total knee arthroplasty. J Arthroplasty. 2010;25(4):507-513. doi:10.1016/j.arth.2009.04.018

15. Berger RA, Rosenberg AG, Barden RM, Sheinkop MB, Jacobs JJ, Galante JO. Long-term followup of the Miller-Galante total knee replacement. Clin Orthop Relat Res. 2001;(388):58-67.

16. Forsythe ME, Englund RE, Leighton RK. Unicondylar knee arthroplasty: A cementless perspective. Can J Surg. 2000;43(6):417-424. doi:10.1175/2010JAS3418.1

17. Lombardi A V., Berasi CC, Berend KR. Evolution of Tibial Fixation in Total Knee Arthroplasty. J Arthroplasty. 2007;22(4 SUPPL.):25-29. doi:10.1016/j.arth.2007.02.006

18. Pilliar RM, Lee JM, Maniatopolous C. Observations on the Effect of Movement on Bone Ingrowth into Porous-Surfaced Implants. Clin Orthop Relat Res. 1986;208:108-113.

19. Stulberg SD, Stulberg BN, Hamati Y, Tsao A. Failure mechanisms of metal-backed patellar components. Clin Orthop Relat Res. 1988;17(236):88-105.

doi:10.1177/036354658901700579

20. Benson LC, DesJardins JD, Harman MK, LaBerge M. Effect of Stair Descent Loading on Ultra-High Molecular Weight Polyethylene Wear in a Force-Controlled Knee Simulator. Vol 216. SAGE PublicationsSage UK: London, England; 2002. doi:10.1243/095441102321032201

21. Bhimji S, Meneghini RM. Micromotion of Cementless Tibial Baseplates Under Physiological Loading Conditions. J Arthroplasty. 2012;27(4):648-654. doi:10.1016/j.arth.2011.06.010

22. Small SR, Rogge RD, Malinzak RA, et al. Micromotion at the tibial plateau in primary and revision total knee arthroplasty: fixed versus rotating platform designs. Bone Jt Res. 2016;5(4):122-129. doi:10.1302/2046-3758.54.2000481

23. Peters CL, Craig MA, Mohr RA, Bachus KN. Tibial Component Fixation With Cement. Clin 
Orthop Relat Res. 2003;409(409):158-168. doi:10.1097/01.blo.0000058638.94987.20

373

374

375

376

377

378

379

380

381

382

383

384

385

386

387

388

389

390

391

392

393

394

395

396

397

398

399

400

401

402

403

404

405

406

407

408

24. Rho JY, Hobatho MC, Ashman R. Relations of mechanical properties to density and CT numbers in human bone. Med Eng Phys. 1995;17(5):347-355. doi:10.1007/978-94-0100367-4_13

25. Taylor M, Barrett DS, Deffenbaugh D. Influence of loading and activity on the primary stability of cementless tibial trays. J Orthop Res. 2012;30(9):1362-1368.

doi:10.1002/jor.22056

26. Harrysson OLA, Robertsson O, Nayfeh JF. Higher Cumulative Revision Rate of Knee Arthroplasties in Younger Patients with Osteoarthritis. Clin Orthop Relat Res. 2004;(421):162-168.

27. Robertsson O, Knutson K, Lewold S, Lidgren L. The Swedish Knee Arthroplasty Register 1975-1997: An update with special emphasis on 41,223 knees operated on in 1988-1997. Acta Orthop Scand. 2001;72(5):503-513. doi:10.1080/000164701753532853

28. Kwong LM, Nielsen ES, Ruiz DR, Hsu AH, Dines MD, Mellano CM. Cementless total knee replacement fixation: A contemporary durable solution-affirms. Bone Jt J. 2014;96B(11):87-92. doi:10.1302/0301-620X.96B11.34327

29. Hu B, Chen Y, Zhu H, Wu H, Yan S. Cementless Porous Tantalum Monoblock Tibia vs Cemented Modular Tibia in Primary Total Knee Arthroplasty: A Meta-Analysis. J Arthroplasty. 2017;32(2):666-674. doi:10.1016/j.arth.2016.09.011

30. De Martino I, D’Apolito R, Sculco PK, Poultsides LA, Gasparini G. Total Knee Arthroplasty Using Cementless Porous Tantalum Monoblock Tibial Component: A Minimum 10-Year Follow-Up. J Arthroplasty. 2016;31(10):2193-2198. doi:10.1016/j.arth.2016.03.057

31. Niemeläinen M, Skyttä ET, Remes V, Mäkelä K, Eskelinen A. Total knee arthroplasty with an uncemented trabecular metal tibial component: A registry-based analysis. J Arthroplasty. 2014;29(1):57-60. doi:10.1016/j.arth.2013.04.014

32. Choy WS, Yang DS, Lee KW, Lee SK, Kim KJ, Chang SH. Cemented versus cementless fixation of a tibial component in LCS mobile-bearing total knee arthroplasty performed by a single surgeon. J Arthroplasty. 2014;29(12):2397-2401. doi:10.1016/j.arth.2014.03.006

33. Fernandez-Fairen M, Hernández-Vaquero D, Murcia A, Torres A, Llopis R. Trabecular metal in total knee arthroplasty associated with higher knee scores: A randomized controlled trial. Clin Orthop Relat Res. 2013;471(11):3543-3553. doi:10.1007/s11999013-3183-4

34. Ghalayini SRA, Helm AT, McLauchlan GJ. Minimum 6year results of an uncemented trabecular metal tibial component in total knee arthroplasty. Knee. 2012;19(6):872-874. doi:10.1016/j.knee.2012.05.001

35. Bobyn JD, Poggie RA, Krygier JJ, et al. Clinical Validation of a Structural Porous Tantalum 
Biomaterial for Adult Reconstruction. J Bone Jt Surg. 2004;86-A(Suppl 2):123-129.

36. Behery OA, Kearns SM, Rabinowitz JM, Levine BR. Cementless vs Cemented Tibial Fixation in Primary Total Knee Arthroplasty. J Arthroplasty. 2017;32(5):1510-1515. doi:10.1016/j.arth.2016.12.023

37. Bhimji S, Meneghini RM. Micromotion of cementless tibial baseplates: Keels with adjuvant pegs offer more stability than pegs alone. J Arthroplasty. 2014;29(7):1503-1506. doi:10.1016/j.arth.2014.02.016

38. Chong DYR, Hansen UN, Amis AA. Analysis of bone-prosthesis interface micromotion for cementless tibial prosthesis fixation and the influence of loading conditions. J Biomech. 2010;43(6):1074-1080. doi:10.1016/j.jbiomech.2009.12.006

39. Dempsey AJ, Finlay JB, Bourne RB, Rorabeck CH, Scott MA, Millman JC. Stability and anchorage considerations for cementless tibial components. J Arthroplasty. 1989;4(3):223-230. doi:10.1016/S0883-5403(89)80018-X

40. Efe T, Figiel J, Danek S, Tibesku CO, Paletta JRJ, Skwara A. Initial stability of tibial components in primary knee arthroplasty. A cadaver study comparing cemented and cementless fixation techniques. Acta Orthop Belg. 2011;77(3):320-328.

41. Kraemer WJ, Harrington IJ, Hearn TC. Micromotion secondary to axial, torsional, and shear loads in two models of cementless tibial components. J Arthroplasty. 1995;10(2):227-235. doi:10.1016/S0883-5403(05)80132-9

42. Matsuda S, Tanner MG, White SE, Whiteside LA. Evaluation of Tibial Component Fixation in Specimens Retrieved at Autopsy. Clin Orthop Relat Res. 1999;363:249-257.

43. Meneghini RM, Daluga A, Soliman M. Mechanical stability of cementless tibial components in normal and osteoporotic bone. J Knee Surg. 2011;24(3):191-196. doi:10.1055/s-0031-1280879

44. Sala M, Taylor M, Tanner KE. Torsional Stability of Primary Total Knee Replacement Tibial Prosetheses: A Biomechanical Study in Cadaveric Bone. J Arthroplasty. 1999;14(5):610615.

45. Walker PS, Hsu H-P, Zimmerman RA. A comparative study of uncemented tibial components. J Arthroplasty. 1990;5(3):245-253. doi:10.1016/S0883-5403(08)80079-4

46. Benzing C, Skwara A, Figiel J, Paletta J. Initial stability of a new cementless fixation method of a tibial component with polyaxial locking screws: a biomechanical in vitro examination. Arch Orthop Trauma Surg. 2016;136(9):1309-1316. doi:10.1007/s00402016-2517-6

47. Crook PD, Owen JR, Hess SR, Al-Humadi SM, Wayne JS, Jiranek WA. Initial Stability of Cemented vs Cementless Tibial Components Under Cyclic Load. J Arthroplasty. 2017;32(8):2556-2562. doi:10.1016/j.arth.2017.03.039 

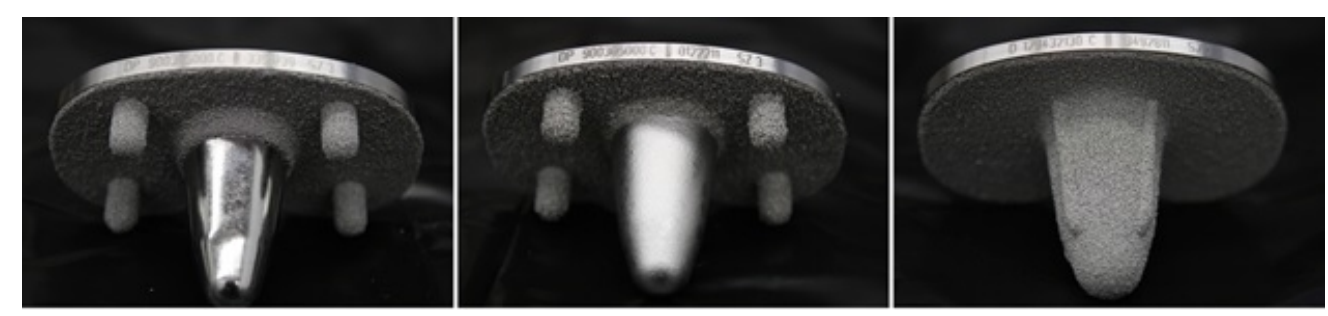

Figure 1: (Left) LCS Duofix cementless tibial tray with sintered bead coating "DuoP", (Center) LCS Duofix cementless tibial component with roughened porous coating "DuoG", (Right) LCS MBT cementless tibial tray

$133 \times 29 \mathrm{~mm}(120 \times 120 \mathrm{DPI})$ 


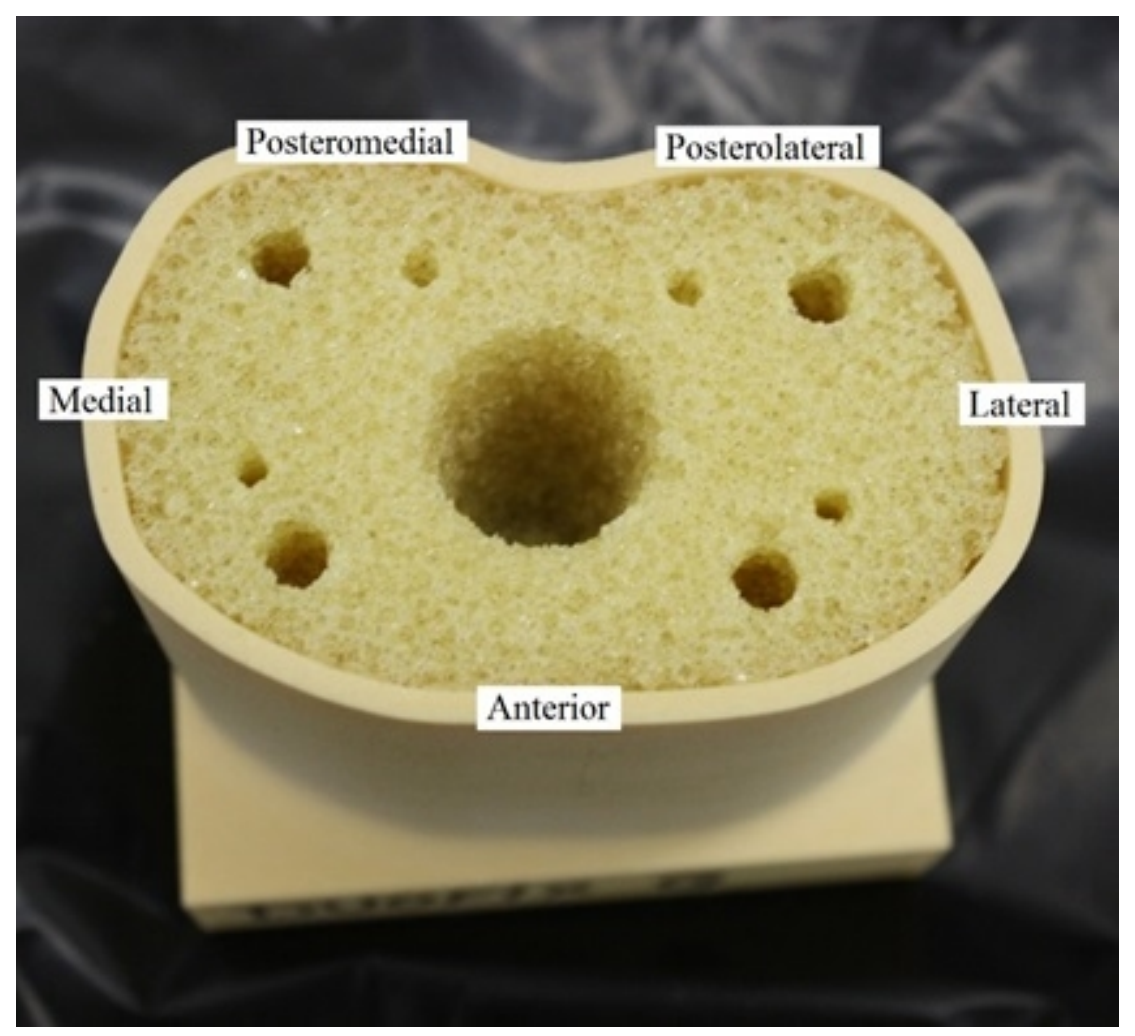

Figure 2: Top view of the two-layered analogue tibial model with cortical foam layer filled with open-cell replicate cancellous foam, with measurement regions.

$89 \times 82 \mathrm{~mm}(120 \times 120 \mathrm{DPI})$ 


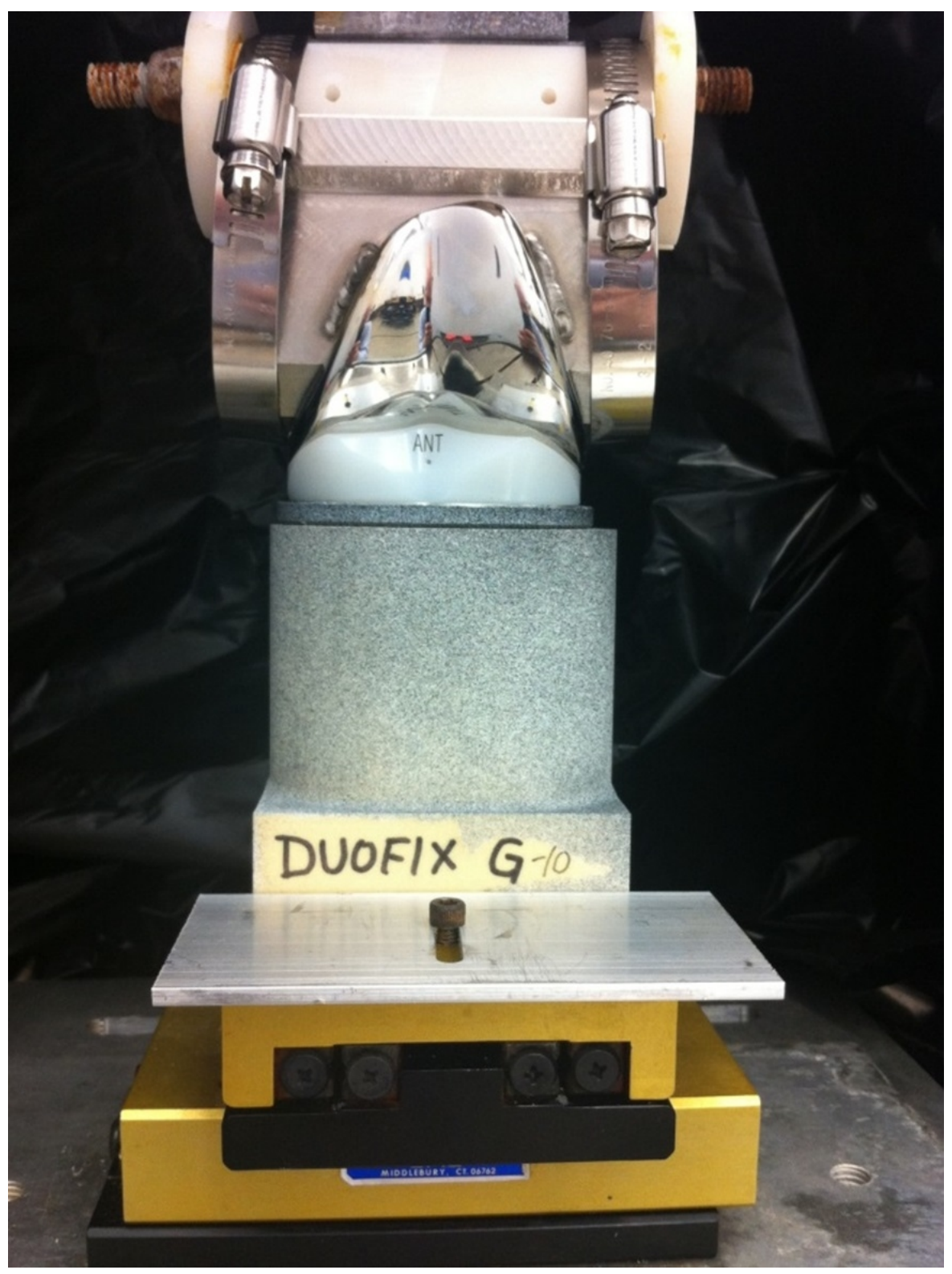

Figure 3: Loading setup for stair descent micromotion analysis in foam model. $185 \times 248 \mathrm{~mm}(120 \times 120 \mathrm{DPI})$ 


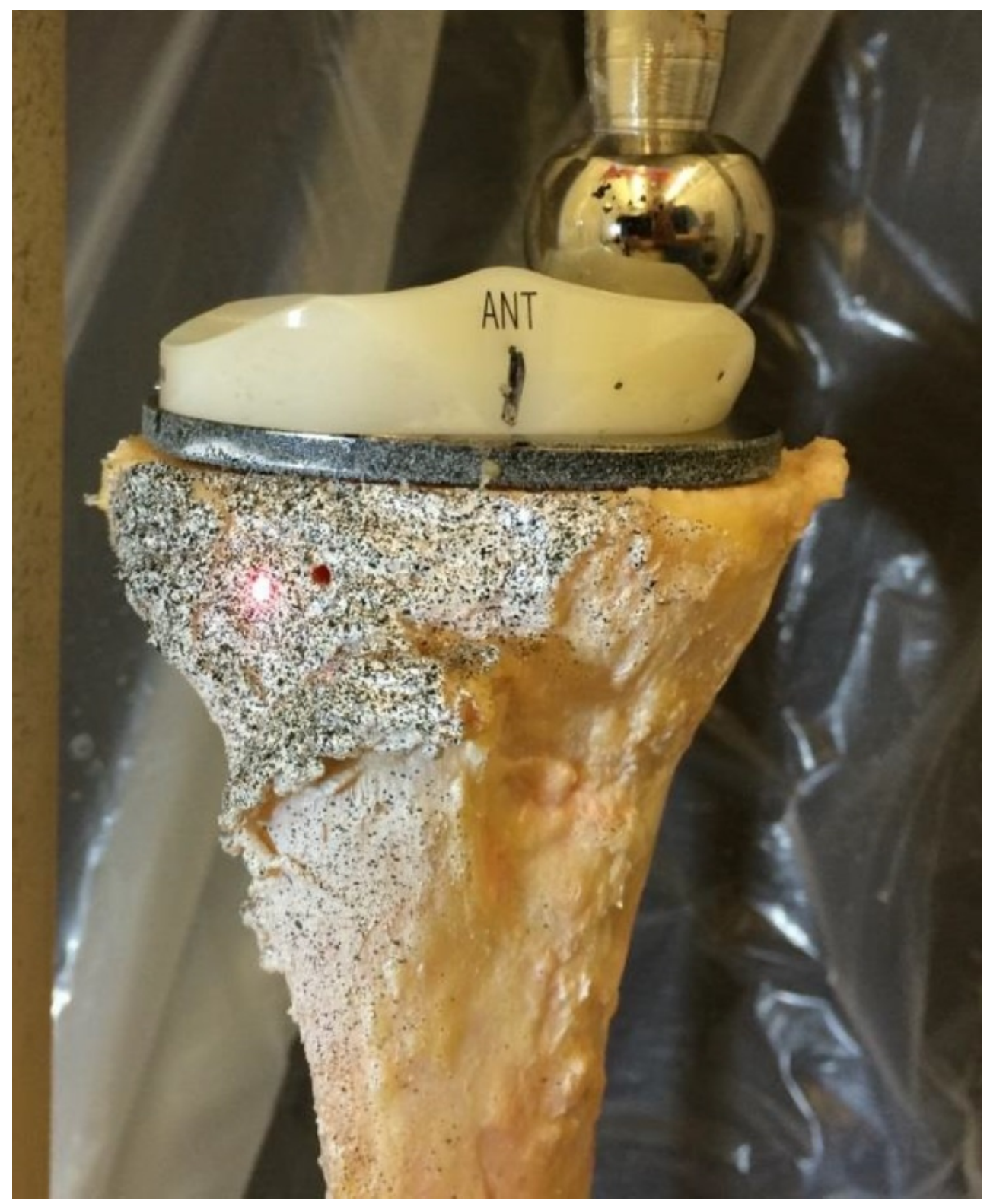

Figure 4: Speckled tibia specimen and tibial tray with loading sphere applied to a single condyle on the rotating platform polyethylene bearing.

$157 \times 193 \mathrm{~mm}(120 \times 120 \mathrm{DPI})$ 


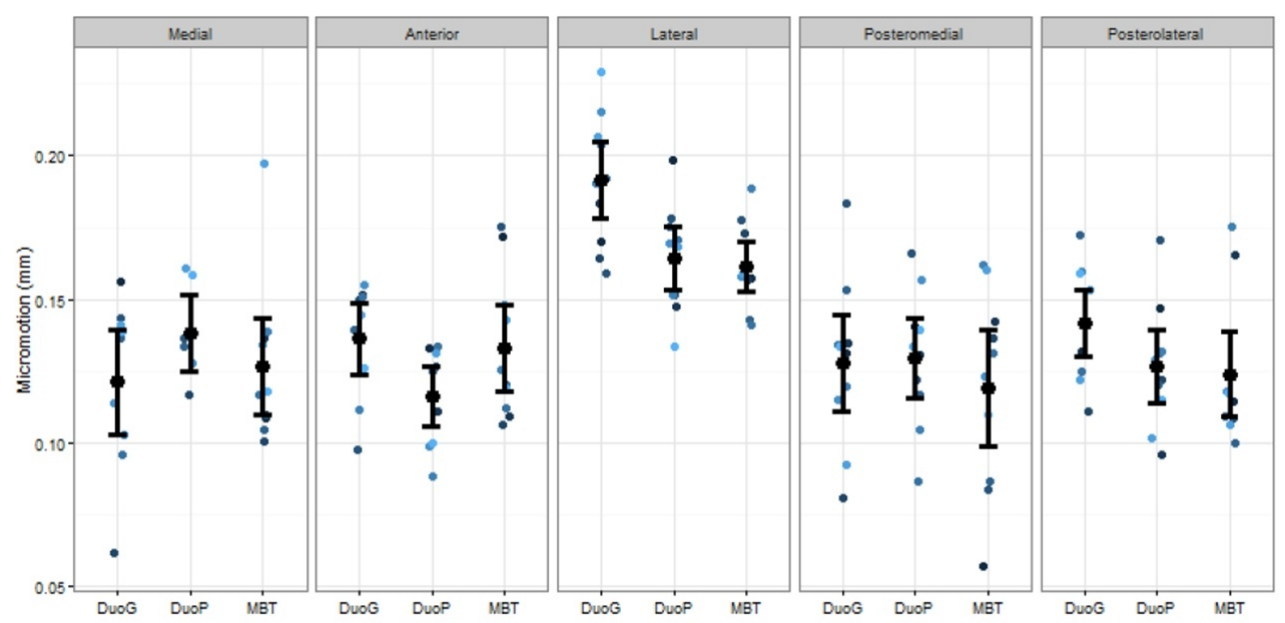

Figure 5: Relative micromotion between implanted tibial tray and foam tibia model during stair descent loading. The estimated mean micromotion and associated $95 \% \mathrm{CI}$ are included.

$275 \times 140 \mathrm{~mm}(120 \times 120 \mathrm{DPI})$ 


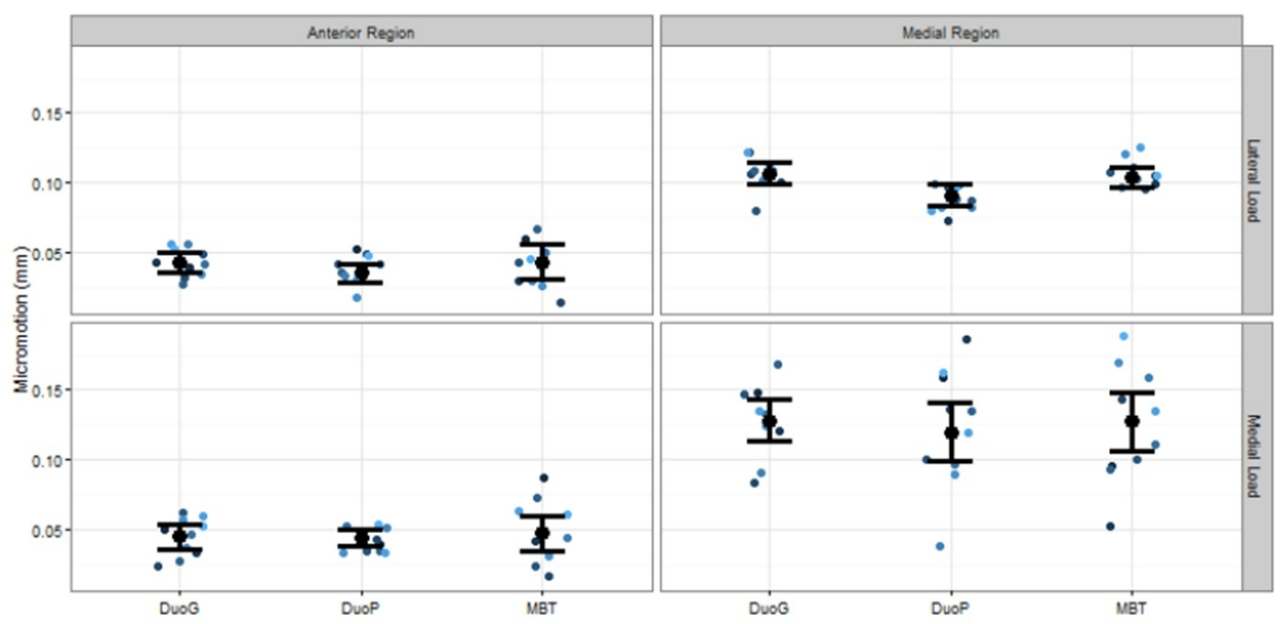

Figure 6: Relative micromotion between implanted tibial tray and foam tibia model during condylar liftoff testing. The estimated mean micromotion and associated 95\% CI are included.

$299 \times 151 \mathrm{~mm}(120 \times 120 \mathrm{DPI})$ 


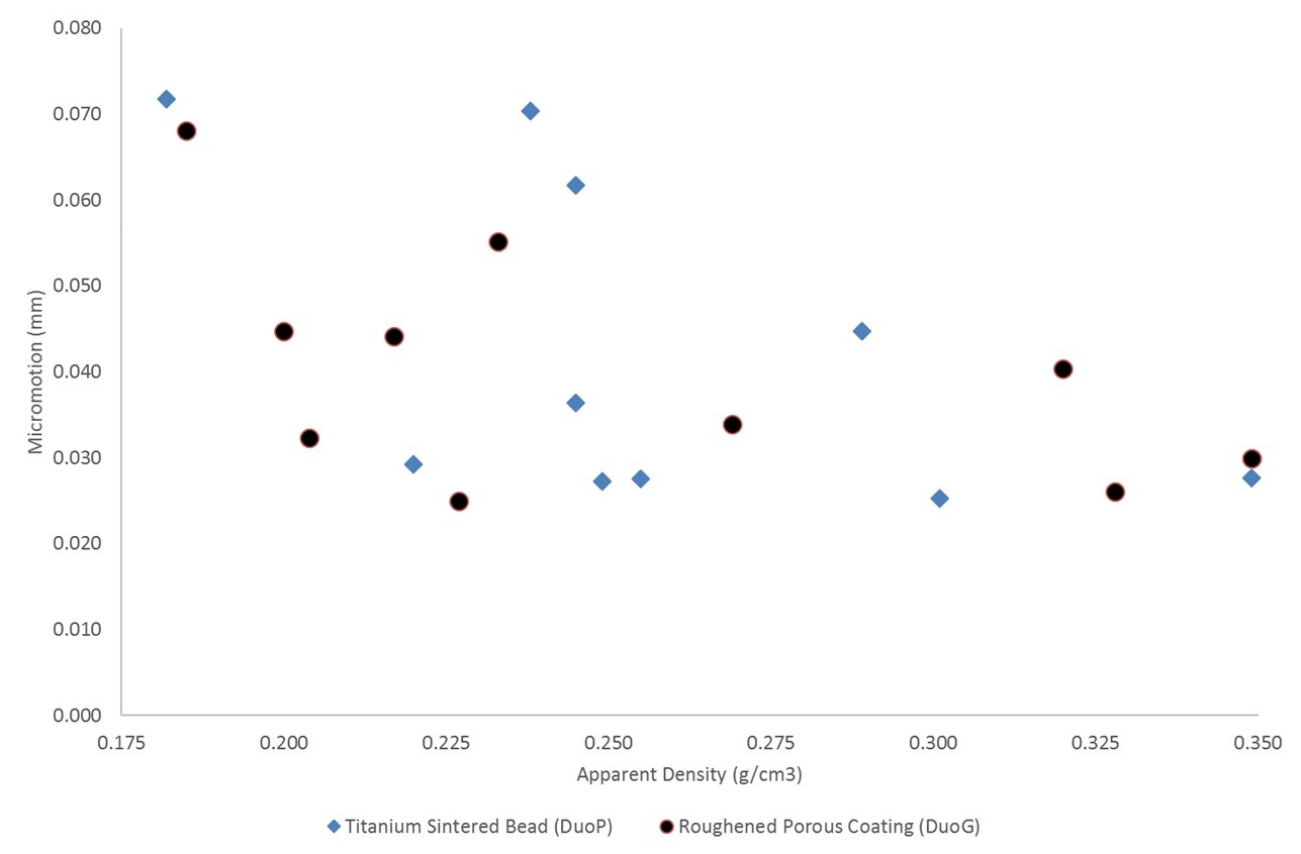

Figure 7: Apparent density versus peak micromotion at 3000 cycles for implanted cadaveric tibias in liftoff testing. A downward trend in micromotion is apparent in both implant designs as specimen density increases.

$272 \times 178 \mathrm{~mm}(120 \times 120 \mathrm{DPI})$ 